\title{
Field alignment of bent-core smectic liquid crystals for analog optical phase modulation
}

\author{
Y. Shen, ${ }^{1}$ L. Goodhew,${ }^{1}$ R. Shao, ${ }^{1}$ M. Moran, ${ }^{2}$ E. Korblova,${ }^{2}$ D. M. Walba,${ }^{2}$ N. A. Clark, ${ }^{1}$ \\ J. E. Maclennan, ${ }^{1}$ and P. Rudquist ${ }^{3, a)}$ \\ ${ }_{1}^{1}$ Department of Physics and Soft Materials Research Center, University of Colorado, \\ Boulder, Colorado 80309, USA \\ ${ }^{2}$ Department of Chemistry and Biochemistry and Soft Materials Research Center, \\ University of Colorado, Boulder, Colorado 80309, USA \\ ${ }^{3}$ Microtechnology and Nanoscience, Chalmers University of Technology, 41296 Göteborg, Sweden
}

(Received 16 January 2015; accepted 30 March 2015; published online 11 May 2015)

\begin{abstract}
A general method for aligning bent-core smectic liquid crystal materials is described. Alternating electric fields between interdigitated electrodes patterned on one cell surface create torques on the liquid crystal that result in uniform "bookshelf" orientation of the smectic layers. The aligned cell can then be driven in the conventional way by applying an electric field between all of the stripe electrodes connected together and a monolithic electrode on the other cell surface. Fast, analog, optical phase-only modulation is demonstrated in a device containing a polar, bent-core $\operatorname{SmAP}_{\mathrm{F}}$ material aligned using this technique. (C) 2015 AIP Publishing LLC.
\end{abstract}

[http://dx.doi.org/10.1063/1.4919805]

The 1996 renaissance of interest in so-called "banana" liquid crystals (LCs), a class of mesogens with strongly bent molecular cores ${ }^{1,2}$ has led to the discovery and exploration of a wealth of exotic structural and behavioral phenomena., Bent-core mesogens exhibit a wide variety of phases with different symmetries, unprecedentedly rich and complex optical textures, and new functionalities that are not found in LC phases of rod-like molecules. ${ }^{5-10}$ Of particular interest is the recently discovered $\mathrm{SmAP}_{\mathrm{F}}$ phase, ${ }^{11}$ a non-tilted (orthogonal), achiral, biaxial, and spontaneously polar fluid smectic. The electrooptic response of the $\mathrm{SmAP}_{\mathrm{F}}$ phase in the bookshelf layer geometry (with the layers oriented uniformly, perpendicular to the plates of the cell), in which an applied electric field coupling to the spontaneous polarization changes the effective birefringence without affecting the director orientation, offers the promise of sub-millisecond, analog, phase-only optical modulation devices with tunable phase shifts of several waves, a modulation depth that has not been realized previously. However, most bent-core smectics, including $\mathrm{SmAP}_{\mathrm{F}}$ phase materials, cannot be uniformly aligned in cells using conventional techniques such as rubbed surface alignment layers, a drawback that has impeded the development of any electrooptic devices based on bent-core LCs. While mechanical shearing has been shown to produce some alignment of banana materials, this procedure is relatively complicated to implement. ${ }^{12}$ In this letter, we describe a general field alignment method that can be used to align a variety of polar and paraelectric liquid crystals, including both bent-core and rod-shaped materials. This technique is employed to achieve homogeneous bookshelf alignment over large areas of $\mathrm{SmAP}_{\mathrm{F}}$ cells, allowing us to demonstrate the unique, phase-only optical modulation capabilities of this liquid crystal phase.

a)per.rudquist@chalmers.se
In the $\mathrm{SmAP}_{\mathrm{F}}$ phase, the spontaneous polarization $\mathbf{P}_{s}$ is oriented in the bow plane of the molecules, perpendicular to the molecular long axis or director $\mathbf{n}$. An electric field $\mathbf{E}$ applied normal to $\mathbf{n}$ couples to $\mathbf{P}_{s}$ and causes the optical indicatrix to rotate about $\mathbf{n}$, while keeping the director fixed in space. Light incident along the applied field direction and polarized perpendicular to $\mathbf{n}$ consequently encounters a fielddependent refractive index $n_{\perp}$ that depends on the orientation of $\mathbf{P}_{s}$ as

$$
n_{\perp}=\frac{n_{1} n_{2}}{\sqrt{n_{1}^{2} \sin ^{2} \phi+n_{2}^{2} \cos ^{2} \phi}},
$$

where $n_{1}$ and $n_{2}$ are the refractive indices in the smectic plane, perpendicular and parallel to $\mathbf{P}_{s}$, respectively, and $\phi$ is the angle between $\mathbf{P}_{s}$ and the direction of the light. In an ideal $\mathrm{SmAP}_{\mathrm{F}}$ optical phase modulator, the smectic layers would be uniformly aligned in the bookshelf geometry and an electric field applied normal to the plane of the cell would be used to achieve optical phase modulation over a range limited only by the thickness of the LC layer. Such a device could alternatively be used for amplitude modulation by inserting it between crossed polarizers, with the layer normal oriented at $45^{\circ}$ from the polarizer axes. The remanent inplane birefringence of the field-off state could be compensated using birefringent film.

Since $\operatorname{SmAP}_{\mathrm{F}}$ materials have large spontaneous polarization (typically several hundred $\left.\mathrm{nC} / \mathrm{cm}^{2}\right),{ }^{11}$ space charge effects dominate in determining their director configuration and electrooptic response. In a bookshelf geometry cell, the polarization in the absence of applied field is essentially uniform and the LC reorients as a uniform block in response to an applied voltage, ${ }^{11,13}$ allowing access to all orientations of the optical indicatrix about its long axis. ${ }^{14,15}$ The cell has an analog electrooptic response with memory, i.e., with no power dissipation until the next state is written. ${ }^{16}$ The absence of electric field in the LC layer in the steady state prevents the 
accumulation of ions, so that the device does not require dc balance. $\mathrm{SmAP}_{\mathrm{F}}$ materials have short field response times comparable to those of $\mathrm{SmC}^{*}$ devices, switching in tens of microseconds rather than the milliseconds typical of nematicbased modulators.

W586, the prototype polar smectic A mesogen and the main $\mathrm{SmAP}_{\mathrm{F}}$ material used in this study, ${ }^{11}$ belongs to a family of bent-core molecules with a polar termination on one end and a siloxane-terminated tail on the other as shown in Fig. 1(a). On cooling from the isotropic, W586 enters a uniaxial, paraelectric SmA phase followed by a broad $\operatorname{SmAP}_{\mathrm{F}}$ phase, below which the material crystallizes. The $\operatorname{SmAP}_{\mathrm{F}}$ phase is optically biaxial, with $\delta n=n_{2}-n_{1} \approx 0.02$. All of the $\mathrm{SmAP}_{\mathrm{F}}$ mesogens in this family show field alignment and phase modulation behavior similar to W586, described in detail below.

Sample cells, illustrated schematically in Fig. 1(b), were manufactured in the Nanofabrication Laboratory at the Chalmers University of Technology. The top surface of the cell had a single, square indium tin oxide (ITO) electrode, while the bottom had interdigitated electrodes created using optical lithography and wet etching. In cells $2 \mu \mathrm{m}(9 \mu \mathrm{m})$ thick, the width of the ITO stripes and the gap between them were both nominally $3 \mu \mathrm{m}(15 \mu \mathrm{m})$. The total active electrode area was $3 \mathrm{~mm} \times 3 \mathrm{~mm}$. The cell thickness was maintained using silica spheres dispersed in glue lines near the edges of the glass substrates. ${ }^{17}$

In a typical alignment experiment, the cell was capillaryfilled at elevated temperature, with the liquid crystal in the isotropic phase. The cell was then cooled (at a rate of $-1^{\circ}$ $\mathrm{C} / \mathrm{min}$ ) from the isotropic to the uniaxial SmA phase, where it displayed random focal conic texture (Fig. 2(a)). An ac voltage was then applied between the interdigitated electrodes in order to induce uniform bookshelf alignment. Applying a square wave voltage $(E \approx 5 \mathrm{~V} / \mu \mathrm{m}$ at $1 \mathrm{~Hz})$ for a few seconds in the SmA phase was typically enough to align the entire active area of the cell uniformly in the bookshelf geometry with the layer normal $\mathbf{z}$ parallel to the stripes, as shown in

(a)

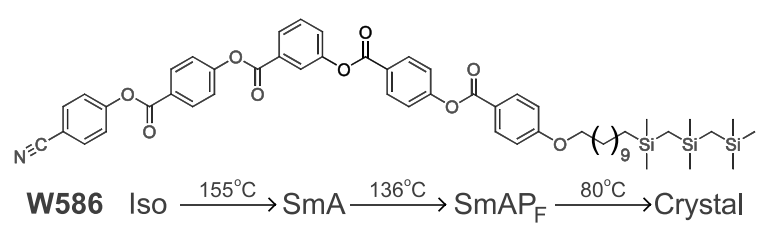

(b)

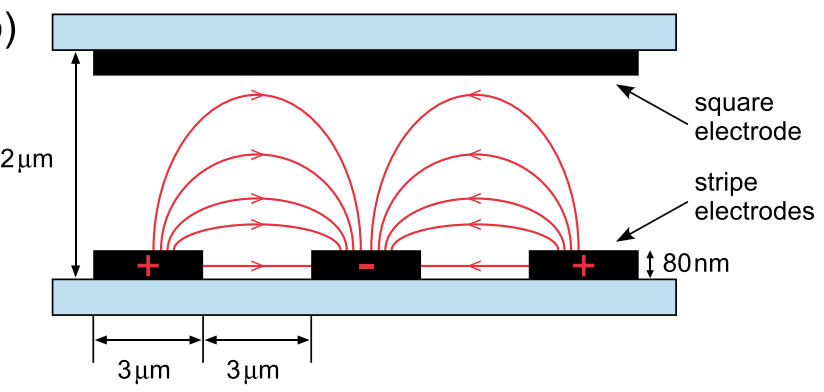

FIG. 1. W586 structure and sample cell geometry. (a) Molecular structure and phase sequence of the bent-core liquid crystal W586. (b) The upper glass plate of the cell has a single, square ITO electrode, while the lower plate has interdigitated electrodes for field alignment. The electrode thickness is greatly exaggerated here.

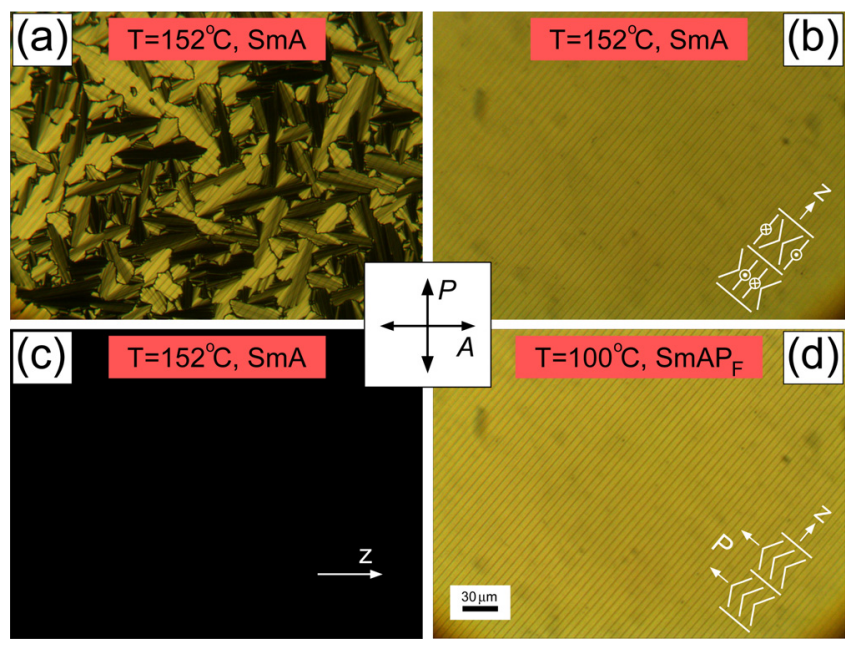

FIG. 2. Alignment of the bent-core mesogen W586 in a $2 \mu$ m thick cell between crossed polarizer and analyzer before and after field application. (a) Random focal-conic texture in a virgin cell cooled from the isotropic to the $\mathrm{SmA}$ phase. (b) After field alignment, the smectic layer normal $\mathbf{z}$ is oriented uniformly, parallel to the electrodes. When the electrodes are oriented at $45^{\circ}$ to the polarizers, a homogeneous birefringence color slightly modulated by the stripe electrodes but with no alignment defects is observed. (c) When the electrodes are parallel to either polarizer, a perfect dark state is achieved. (d) Alignment is maintained after cooling into the $\operatorname{SmAP}_{\mathrm{F}}$ phase.

Figs. 2(b) and 2(c). This alignment was retained after the field was removed and was preserved on subsequent cooling to the $\mathrm{SmAP}_{\mathrm{F}}$ phase (Fig. 2(d)).

Field-induced alignment was studied over a range of temperature and applied field conditions, as follows. (i) Temperature. When a $5 \mathrm{~V} / \mu \mathrm{m}$ square-wave alignment field at $1 \mathrm{~Hz}$ was applied while cooling from the isotropic into the SmA phase at a rate of $-1^{\circ} \mathrm{C} / \mathrm{min}$, or while keeping the sample at a constant temperature just below the transition to the SmA phase, a defect-free bookshelf structure was obtained within a few seconds. When the electric field was applied only at lower temperature in the SmA phase (close to the $\mathrm{SmAP}_{\mathrm{F}}$ transition), or with the sample already in the $\mathrm{SmAP}_{\mathrm{F}}$ phase, alignment could also be induced but only with a significantly higher applied voltage and the alignment in this case was never as good as that obtained at higher temperature, where the smectic layers are relatively soft, ${ }^{18}$ there is weaker surface anchoring of the layers, and lower viscosity facilitates flow. Although the spontaneous polarization in the $\mathrm{SmAP}_{\mathrm{F}}$ phase is higher than the induced polarization in the SmA phase, the applied field in the $\mathrm{SmAP}_{\mathrm{F}}$ phase is at least partly screened ${ }^{13}$ and the layers are more rigid. The higher voltages required to induce alignment at lower temperatures tended to damage the cell before uniform layer orientation was obtained. (ii) Waveform. While a $1 \mathrm{~Hz}$ square-wave voltage applied at high temperatures rapidly led to uniform alignment of the sample, a sine or triangle wave with the same amplitude was less effective. Increasing the frequency of the applied voltage, for example, to 10 or $100 \mathrm{~Hz}$, resulted in cells that were noticeably less well aligned the higher the frequency. For $f \geq 100 \mathrm{~Hz}$, there was no observable alignment effect and the alignment field tended to cause short-circuits. The rate and degree of alignment were also found to depend strongly on the magnitude of the applied field. For sufficiently small fields $(E \lesssim 2 \mathrm{~V} / \mu \mathrm{m})$, there was no alignment at all, suggesting that 
there is a minimum field strength below which rearrangement of the smectic layers does not readily occur. Above this threshold, the time required to align the cell decreased and the final alignment quality improved as the applied voltage was increased up to about $6 \mathrm{~V} / \mu \mathrm{m}$, where excellent alignment was obtained within a few seconds.

Electric field-induced reorientation of smectic layers of rod-like LCs has been studied extensively in cells of chiral $\mathrm{SmA}^{*}$ and $\mathrm{SmC}^{*}$ materials ${ }^{19,20}$ and is well known from the field-mediated straightening of vertical chevrons in $\mathrm{SmC}^{*}$ liquid crystals. ${ }^{21}$ Chen et al. recently demonstrated switching between two stable states-with homeotropic and uniform bookshelf alignment - in a conventional achiral SmA material of rod-like mesogens with positive dielectric anisotropy, using patterned cells similar to those employed here. ${ }^{22}$ Nakata et al. observed local, field-induced, bookshelf alignment of the tilted, polar bent-core $\mathrm{SmC}_{\mathrm{A}} \mathrm{P}_{\mathrm{F}}^{*}$ phase in the gap between two planar electrodes on the same surface of a cell, $^{23}$ and a similar method was used by Kinoshita to align domains of the antiferroelectric $\mathrm{B}_{2}$ banana phase. ${ }^{24}$

In our experiments, the observed field-alignment of the liquid crystal results from coupling between the LC polarization $\mathbf{P}$ (whether induced or spontaneous) and the applied field $\mathbf{E}$. In both the $\mathrm{SmA}$ and $\mathrm{SmAP}_{\mathrm{F}}$ phases, when $\mathbf{P}$ is not parallel to $\mathbf{E}$ the layers experience a non-zero torque which, when sufficiently large, reorients the smectic layers to make the induced polarization parallel to the applied field everywhere. Although the field generated by the stripe electrodes is on average parallel to the bounding plates of the cell, it is spatially inhomogeneous and the field lines are curved in the cell interior, as indicated in Fig. 1(b). The minimum energy configuration that has $\mathbf{P}$ parallel to $\mathbf{E}$ everywhere can be achieved only if the smectic layer normal $\mathbf{z}$ is oriented parallel to the stripe electrodes and the layers have homogeneous bookshelf alignment.

A W586 cell that has been field-aligned in the isotropic or SmA phase can be cooled to the $\operatorname{SmAP}_{\mathrm{F}}$ phase with the layer structure preserved and then driven in the conventional way by applying an electric field across the thickness of the cell. This is implemented by connecting the finger electrodes together to make one effective electrode on the bottom plate, opposing the large, single electrode on the top plate. With the cell oriented at $45^{\circ}$ between crossed polarizers, changes in $\Delta n$ with applied voltage are manifested as variations of birefringence color (or of intensity when the incident light is monochromatic). Upon increasing the applied voltage from zero, where $\mathbf{P}_{s}$ is parallel to the substrates $(\phi=\pi / 2)$, to the saturated state, where $\mathbf{P}_{s}$ is normal to the substrates $(\phi=0)$, the sample birefringence, determined using a Berek compensator, increases continuously from $\Delta n=n_{3}-n_{2} \approx 0.9$ to $\Delta n=n_{3}-n_{1} \approx 0.11$, as shown in Fig. 3(a). Since the maximum phase modulation depth is $\delta_{\max }=2 \pi\left(n_{2}-n_{1}\right) d / \lambda$, a full wave of modulation $(\delta=2 \pi)$ would be obtained in a W586 cell of thickness $d=28 \mu \mathrm{m}$ (or $d=14 \mu \mathrm{m}$ in reflective mode). The small, periodic modulation of the cell birefringence visible in Figs. 2 and 3 is a direct result of the presence and absence of ITO on the lower glass plate. This variation in optical appearance is a well-known artefact in both nematic and ferroelectric liquid crystal cells with patterned electrodes that can be

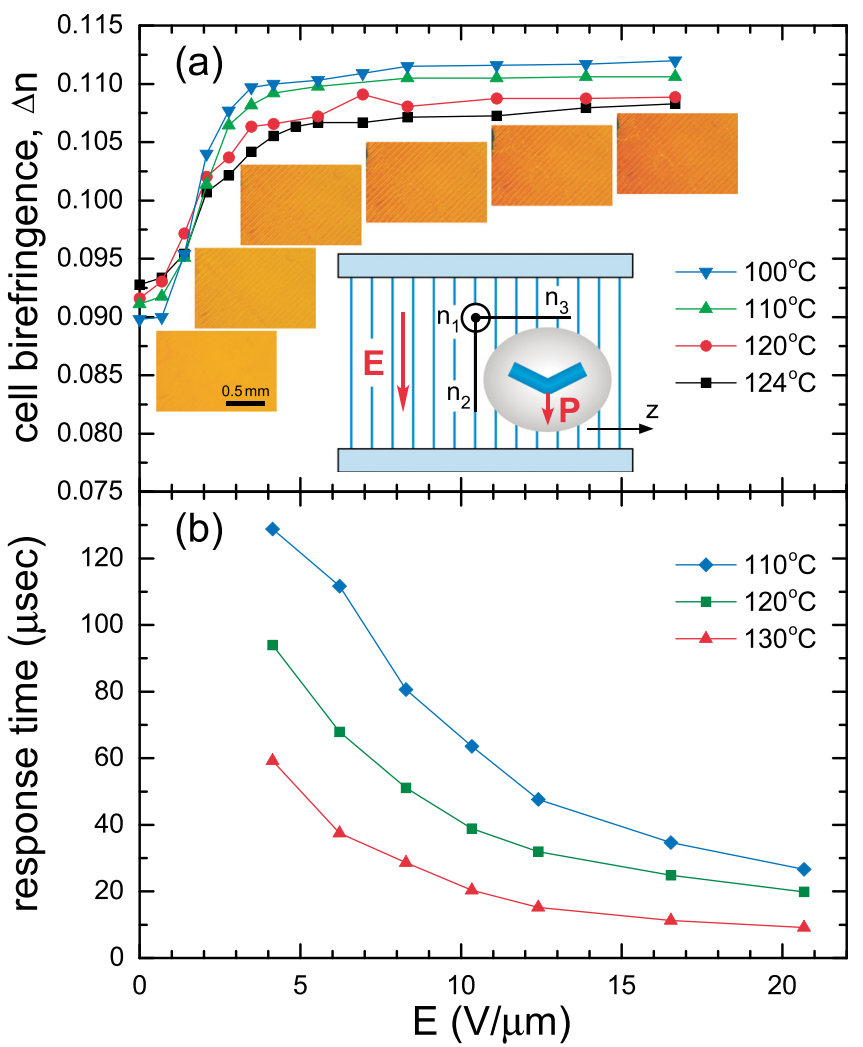

FIG. 3. Electrooptic response of a field-aligned $\mathrm{SmAP}_{\mathrm{F}}$ cell of W586. (a) Birefringence at selected temperatures in the $\operatorname{SmAP}_{\mathrm{F}}$ phase as a function of applied electric field. Inset: The applied field $\mathbf{E}$ couples to the polarization $\mathbf{P}$, inducing rotation of the biaxial indicatrix with principal indices $\left(n_{1}, n_{2}, n_{3}\right)$ about the layer normal $\mathbf{z}$. (b) Response time as a function of applied electric field. The cell thickness is $9 \mu \mathrm{m}$.

eliminated in reflective mode devices by adding a dielectric mirror coating. 25

The switching response times of the aligned $\operatorname{SmAP}_{F}$ phase, derived from the polarization current response to a square-wave driving voltage, ${ }^{26}$ are on the order of tens of microseconds, as shown in Fig. 3(b), and vary inversely with the applied field strength as expected.

In summary, we have demonstrated that the $\mathrm{SmAP}_{\mathrm{F}}$ phase of bent-core liquid crystals can be aligned with perfect bookshelf geometry using in-plane electric fields and then utilized for fast, analog phase modulation of light. The optical modulation range of this device is limited, in principle, only by the cell thickness. By patterning interdigitated electrodes with different orientations in different parts of the cell, carefully designed multi-domain $\mathrm{SmAP}_{\mathrm{F}}$ devices could easily be realized. Smectic devices are somewhat sensitive to mechanical shock but since the driving voltages used to operate the device are of the same magnitude as those needed for alignment; the layer alignment in a damaged cell could be restored simply by heating the device and repeating the field alignment procedure.

Finally, we have established that the technique described here is remarkably versatile and can be used to align ferroelectric and paraelectric smectic liquid crystals of both rodshaped and bent-core mesogens. We have, for example, obtained well-aligned, bookshelf geometry domains of several different chiral phases and materials, including the B2 phase of achiral bent-core materials, the $\mathrm{SmA}^{*}$ and $\mathrm{SmC}^{*}$ phases of both conventional and de Vries-type smectics, and the $\mathrm{SmC}_{\mathrm{A}}^{*}$ 
phase of antiferroelectric smectics. Surprisingly, in-plane electric fields were even found to align the SmA phase of racemic, rod-shaped liquid crystals very well.

This work was supported by the Soft Materials Research Center under NSF MRSEC Grant Nos. DMR-0820579 and DMR-1420736, by NSF Grant Nos. DMR-1008300 and DMR-1306209, and by Swedish Research Council (VR) Grant No. 621-2009-3621.

${ }^{1}$ T. Niori, T. Sekine, J. Watanabe, T. Furukawa, and H. Takezoe, J. Mater. Chem. 6, 1231 (1996).

${ }^{2}$ D. Link, G. Natale, R. Shao, J. Maclennan, N. Clark, E. Korblova, and D. Walba, Science 278, 1924 (1997).

${ }^{3}$ H. Takezoe and Y. Takanishi, Jpn. J. Appl. Phys., Part 1 45, 597 (2006).

${ }^{4}$ R. Reddy and C. Tschierske, J. Mater. Chem. 16, 907 (2006).

${ }^{5}$ R. Reddy and B. Sadashiva, J. Mater. Chem. 14, 310 (2004).

${ }^{6}$ D. Pociecha, E. Gorecka, M. Čepič, N. Vaupotič, K. Gomola, and J. Mieczkowski, Phys. Rev. E 72, 060701 (2005).

${ }^{7}$ K. Gomola, L. Guo, E. Gorecka, D. Pociecha, J. Mieczkowski, K. Ishikawa, and H. Takezoe, Chem. Commun. 2009, 6592-6594.

${ }^{8}$ Y. Shimbo, Y. Takanishi, K. Ishikawa, E. Gorecka, D. Pociecha, J. Mieckowski, K. Gomola, and H. Takezoe, Jpn. J. Appl. Phys., Part 2 45, L282 (2006).

${ }^{9}$ L. Guo, E. Gorecka, D. Pociecha, N. Vaupotič, M. Čepič, R. A. Reddy, K. Gornik, F. Araoka, N. A. Clark, D. M. Walba et al., Phys. Rev. E 84, 031706 (2011).

${ }^{10}$ C. Zhu, R. Shao, R. A. Reddy, D. Chen, Y. Shen, T. Gong, M. A. Glaser, E. Korblova, P. Rudquist, J. E. Maclennan et al., J. Am. Chem. Soc. 134, 9681 (2012).
${ }^{11}$ R. A. Reddy, C. Zhu, R. Shao, E. Korblova, T. Gong, Y. Shen, E. Garcia, M. A. Glaser, J. E. Maclennan, D. M. Walba et al., Science 332, 72 (2011).

${ }^{12}$ A. Rastegar, G. Wulterkens, H. Verscharen, T. Rasing, and G. Heppke, Rev. Sci. Instrum. 71, 4492 (2000).

${ }^{13}$ Y. Shen, T. Gong, R. Shao, E. Korblova, J. E. Maclennan, D. M. Walba, and N. A. Clark, Phys. Rev. E 84, 020701 (2011).

${ }^{14}$ P. Rudquist, J. Lagerwall, M. Buivydas, F. Gouda, S. Lagerwall, N. Clark, J. Maclennan, R. Shao, D. Coleman, S. Bardon et al., J. Mater. Chem. 9, 1257 (1999)

${ }^{15}$ N. Clark, D. Coleman, and J. Maclennan, Liq. Cryst. 27, 985 (2000).

${ }^{16}$ D. Walba, U.S. patent application US 2013/0016295 A1 (2013).

${ }^{17}$ These cells were originally designed for a different experiment, with the inner surfaces coated with the polyimide SE1211 (Nissan Chemicals). This material forces the director field of LCs of many rod-like mesogens to be homeotropic (normal to the surface) but gives random-planar alignment of the bent-core materials studied here.

${ }^{18}$ S. D. Siemianowski, P. D. Brimicombe, S. Jaradat, P. Thompson, W. Bras, and H. F. Gleeson, Liq. Cryst. 39, 1261 (2012).

${ }^{19}$ K. Nakayama, H. Moritake, M. Ozaki, and K. Yoshino, Jpn. J. Appl. Phys., Part 234, L1599 (1995).

${ }^{20}$ I. Dierking, L. Komitov, and S. Lagerwall, Jpn. J. Appl. Phys., Part 2 37, L57 (1998).

${ }^{21}$ W. Hartmann and A. Luyckx-Smolders, J. Appl. Phys. 67, 1253 (1990).

${ }^{22}$ H. Chen, R. Shao, E. Korblova, W. Lee, D. Walba, and N. Clark, Appl. Phys. Lett. 91, 163506 (2007).

${ }^{23}$ M. Nakata, D. Link, F. Araoka, J. Thisayukta, Y. Takanishi, K. Ishikawa, J. Watanabe, and H. Takezoe, Liq. Cryst. 28, 1301 (2001).

${ }^{24}$ Y. Kinoshita, M.S. thesis, Tokyo Institute of Technology, Japan, 1999.

${ }^{25}$ S. Serati and J. Stockley, Proc. SPIE 5894, 180 (2005).

${ }^{26}$ K. Skarp, I. Dahl, S. Lagerwall, and B. Stebler, Mol. Cryst. Liq. Cryst. 114, 283 (1984) 